\title{
Epidemiology, clinical features and outcomes of incident tuberculosis in children in Canada in 2013- 2016: results of a national surveillance study
}

\author{
Shaun K Morris (D) , ${ }^{1,2,3}$ Ryan J P Giroux ${ }_{1}^{2,3}$ Raquel Consunji-Araneta, ${ }^{4}$ \\ Kristoffor Stewart, ${ }^{5}$ Maureen Baikie, ${ }^{6}$ Fatima Kakkar, ${ }^{7}$ David Zielinski, ${ }^{8}$ \\ Alena Tse-Chang, ${ }^{9,10}$ Victoria J Cook, ${ }^{11,12}$ Dina A Fisher, ${ }^{13}$ Marina I Salvadori, ${ }^{14,15}$ \\ Jeffrey M Pernica (D) , ${ }^{16}$ Laura J Sauve, ${ }^{12,17}$ Charles Hui, ${ }^{18}$ Amber Miners, ${ }^{19}$ \\ Gonzalo G Alvarez, ${ }^{20,21}$ Assaad Al-Azem, ${ }^{22,23}$ Victor Gallant, ${ }^{24}$ Barbara Grueger, $^{13}$ \\ Ray Lam, ${ }^{1,2}$ Joanne M Langley, ${ }^{25,26}$ Nicole Radziminski, ${ }^{27}$ Elizabeth Rea, ${ }^{28}$ \\ Sam Wong, ${ }^{9,27}$ lan Kitai ${ }^{1,2}$
}

\begin{abstract}
- Additional supplemental material is published online only. To view, please visit the journal online (http://dx.doi. org/10.1136/archdischild2021-322092)
\end{abstract}

For numbered affiliations see end of article.

Correspondence to Dr Shaun K Morris, Infectious Diseases, The Hospital for Sick Children, Toronto, Canada; shaun.morris@sickkids.ca

Received 22 March 2021 Accepted 5 August 2021 Published Online First 20 August 202

Check for updates

(c) Author(s) (or their employer(s)) 2021. No commercial re-use. See rights and permissions. Published by BMJ.

To cite: Morris SK Giroux RJP, Consunji-

Araneta $\mathrm{R}$, et al.

Arch Dis Child

2021:106:1165-1170.

\begin{abstract}
Purpose Childhood tuberculosis disease is difficult to diagnose and manage and is an under-recognised cause of morbidity and mortality. Reported data from Canada do not focus on childhood tuberculosis or capture key epidemiologic, clinical and microbiologic details. The purpose of this study was to assess demographics, presentation and clinical features of childhood tuberculosis in Canada.

Methods We conducted prospective surveillance from 2013 to 2016 of over 2700 paediatricians plus vertical tuberculosis programmes for incident tuberculosis disease in children younger than 15 years in Canada using the Canadian Paediatric Surveillance Program (CPSP).
\end{abstract}

Results In total, 200 cases are included in this study. Tuberculosis was intrathoracic in 183 patients of whom $86 \%$ had exclusively intrathoracic involvement. Central nervous system tuberculosis occurred in 16 cases (8\%). Fifty-one per cent of cases were hospitalised and 11 $(5.5 \%)$ admitted to an intensive care unit. Adverse drug reactions were reported in $9 \%$ of cases. The source case, most often a first-degree relative, was known in 73\% of cases. Fifty-eight per cent of reported cases were Canadian-born Indigenous children. Estimated study rates of reported cases (per 100000 children per year) were 1.2 overall, 8.6 for all Indigenous children and 54.3 for Inuit children.

Conclusion Childhood tuberculosis may cause significant morbidity and resource utilisation. Key geographies and groups have very high incidence rates. Elimination of childhood tuberculosis in Canada will require well-resourced community-based efforts that focus on these highest risk groups.

\section{BACKGROUND}

Globally, there were an estimated 10 million new cases and 1.4 million tuberculosis (TB)-related deaths in $2018 .{ }^{1}$ In Canada, there are on average 1800 incident cases of TB disease per year with approximately $3 \%$ of disease occurring in children younger than 15 years. $^{2}$ In Canada, Indigenous peoples and foreign-born individuals are
What is already known on this topic?

$\Rightarrow$ Childhood tuberculosis is under-reported and under-researched but, globally, is a major hidden cause of childhood mortality.

$\Rightarrow$ According to the Public Health Agency of Canada, childhood tuberculosis accounts for about $3 \%$ of all cases in the country.

$\Rightarrow$ National data lacked important clinical, treatment and epidemiological detail including methods of diagnosis that is needed to improve diagnosis, management and control.

\section{What this study adds?}

$\Rightarrow$ This study prospectively surveyed clinicians and programs managing childhood tuberculosis and provides more epidemiologic and clinical detail than published Canadian data.

$\Rightarrow$ There is substantial variation in regional incidence and remarkably high rates of tuberculosis in Indigenous Canadians who accounted for more than half of cases.

$\Rightarrow$ This study demonstrated high rates of hospitalisation, central nervous system infection with intensive care unit admission and a substantial rate of adverse drug reactions.

disproportionately affected by $\mathrm{TB} .{ }^{3}$ Compared with adult TB, childhood TB disease is more difficult to diagnose, presents unique management challenges ${ }^{45}$ and is an under-recognised cause of morbidity. ${ }^{67}$

TB is a reportable disease at the federal and provincial/territorial levels in Canada. However, available data do not capture important details including the type of disease, use and results of diagnostic tests, rates of culture confirmation and hospitalisation, follow-up data, rates of adverse drug reactions (ADRs), rates in specific childhood populations and whether source cases were identified. To address these gaps, we conducted a multiyear prospective surveillance study of incident TB 
disease in children under the age of 15 in Canada using the Canadian Paediatric Surveillance Program (CPSP). Additional pertinent background information is given in the online supplemental appendix.

\section{METHODS}

The CPSP, a joint project of the Public Health Agency of Canada (PHAC) and the Canadian Paediatric Society, conducts active monthly national surveillance of over 2700 participant paediatricians and paediatric subspecialists for certain childhood disorders. For this study, we also included non-paediatricians from the TB programmes of Alberta, Saskatchewan, Nunavut, the Northwest Territories and British Columbia. We aimed to identify incident cases of childhood TB between October 2013 and September 2016 and follow-up every 6 months until 6 months post-treatment completion. Pertinent data from the TB programme at Hôpital Sainte-Justine in Montréal, Québec, were retrospectively obtained in May 2017 outside of the CPSP platform. The protocol (https:/wwwcpspcpsca/uploads/studies/ childhood-TB-protocolpdf.) initially used existing international case definitions for the diagnosis of intrathoracic childhood TB, ${ }^{8}$ however, revised criteria for intrathoracic TB were published in 2015 so we reviewed and reclassified all cases as confirmed or unconfirmed by consensus adjudication by three authors (SKM, RJPG, IK) according to the new criteria. ${ }^{9}$

We calculated population-based age-specific rates of reported cases using 2016 population data ${ }^{10}$; for those $<1$ year of age we used number of live births. Due to potential under-reporting, rates presented here represent the lowest possible estimate of incidence of childhood TB for specific groups. Rates for Indigenous populations were calculated using denominators from Statistics Canada Aboriginal Population Profile. ${ }^{11}$ Details of study's ethics approvals are provided in the online supplemental appendix.

This work was supported by the Public Health Agency of Canada through the CPSP and a grant from Paediatric Consultants, Hospital for Sick Children.

\section{FINDINGS}

From October 2013 to September 2016, an average of 2536 monthly reporting forms were sent by CPSP and response rates averaged $81 \%$. The number reported and reasons for exclusions are shown in figure 1 and 200 cases met the study case definition. Eighty cases (40\%) were microbiologically confirmed and $116(58 \%)$ were categorised as unconfirmed. The remaining 4 cases were categorised as presumed or difficult to classify. Sixty-six (36\%) and 20 (53\%)of intrathoracic and extrathoracic cases, respectively, were microbiologically confirmed.

The calculated minimum overall rate of reported cases of childhood TB during the study period was 1.2 per 100000 per year compared with an approximate notification rate to PHAC of 1.6/100 000.$^{11}$ Comparisons with data reported by the PHAC are shown in the online supplemental appendix. Key demographics of cases reported to CPSP are shown in table 1. Reports by age group were 2.4 per 100000 live births in children under 1 and $1.8,0.6$ and 0.9 per 100000 population for the age groups 1-4, 5-9 and 10-14 years, respectively. Most cases $(\mathrm{n}=116,58 \%)$ occurred in Canadian-born Indigenous children, the majority of whom were identified as First Nations (67\%) or Inuit $(30 \%)$. The reported case rate in all Indigenous children was $8.6 / 100000 /$ year with the highest rate being in Inuit children (54.3/100000/year) (table 2).

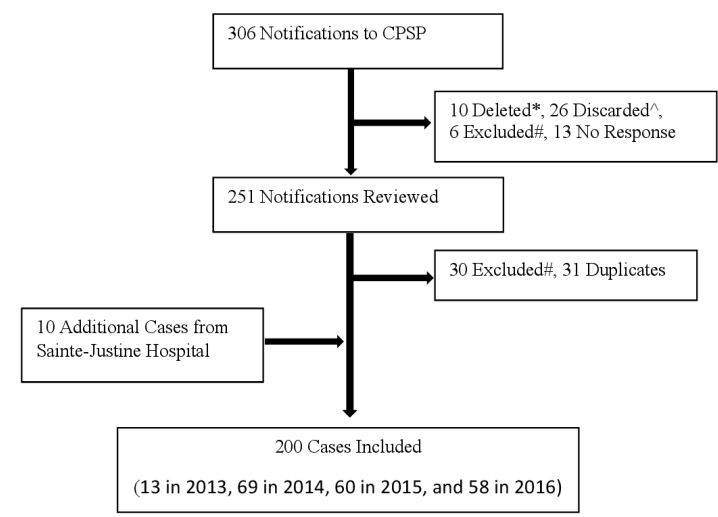

*Deleted refers to case notifications that were deleted by the CPSP primarily for clerical reasons

$\wedge$ Discarded refers to case notifications that the reporting physician or healthcare professional was unable to complete the questionnaire, often because they no longer followed the patient or could not recall the case

\#Excluded refers to case notifications that were noted to not meet inclusion criteria. When excluded by the CPSP this was primarily due to age $15+$ years. When excluded by the Study Investigators, there were multiple demographic and clinical exclusions.

${ }^{\circ}$ No Response refers to case notifications that were sent questionnaires and reminders but did not return a questionnaire or correspond further with the CPSP

$\supseteq$ DDuplicates were noted either by the CPSP program or by comparison of case demographics (sex, birth date, province/territory of residence)

Figure 1 Flowchart of cases notified, excluded and included. CPSP Canadian Paediatric Surveillance Program.

Forty-three (22\%) of reported cases were in Canadianborn non-Indigenous children. Thirty children (15\%) were born outside of Canada of whom 13 (43\%) were born in subSaharan Africa. Among the foreign-born, Africa was the most common continent, and the Philippines and India were the most common countries of birth (table 1). The rates of reported cases for foreign-born and Canadian-born children with at least one foreign-born parent were 2.7 and 1.4 per 100000 , respectively. Of Canadian-born non-Indigenous children, 14 (26\%) travelled to an endemic country.

Most cases resided in Manitoba $(n=62,31 \%)$ followed by Ontario $(n=32,16 \%)$, Québec $(n=28,14 \%)$ and Nunavut

Table 1 Demographic features of cases

\begin{tabular}{|lll}
\hline & Number ( $\mathbf{n})$ & $\%$ \\
\hline Age (N=200) & & \\
\hline$<1$ & 28 & 14 \\
\hline 1 to 4 & 82 & 41 \\
\hline 5 to 9 & 36 & 18 \\
\hline $10+$ & 54 & 27 \\
\hline Sex (N=196) & & \\
\hline Male & 99 & 50.5 \\
\hline Female & 97 & 49.5 \\
\hline Ethnicity (N=200) & & \\
\hline Canadian born, Non-Indigenous & 43 & 21.50 \\
\hline Canadian born, Indigenous, total & 116 & 58 \\
\hline Canadian born, First Nations & $(78)$ & $(39)$ \\
\hline Canadian born, Inuit & $(35)$ & $(17.5)$ \\
\hline Canadian born, other & $<5$ & $n / a$ \\
\hline Canadian born, Not Specified & 11 & 5.5 \\
\hline Non-Canadian born, total (country or region of birth) & 30 & 15 \\
\hline Africa & $(13)$ & $(6.5)$ \\
\hline Philippines & $(10)$ & $(5)$ \\
\hline South Asia & $(5)$ & $(2.5)$ \\
\hline Other & $<5$ & $n / a$ \\
\hline
\end{tabular}


Table 2 Rates of reported case rates by province or territory of residence and Indigenous status

\begin{tabular}{|c|c|c|c|}
\hline & $\begin{array}{l}\text { Rate per } 100 \\
000\end{array}$ & $\begin{array}{l}0-14 \text { Population } \\
\text { size }\end{array}$ & Cases (n) \\
\hline \multicolumn{4}{|l|}{ Canada $(\mathrm{N}=200)$} \\
\hline Canada (N=200) & 1.2 & 5778929 & 200 \\
\hline \multicolumn{4}{|l|}{ Province/territory of residence } \\
\hline British Columbia & 0.4 & 691495 & 8 \\
\hline Alberta & 0.9 & 764638 & 21 \\
\hline Saskatchewan & 3.1 & 213796 & 20 \\
\hline Manitoba & 8.5 & 243534 & 62 \\
\hline Ontario & 0.5 & 2221986 & 32 \\
\hline Québec & 0.7 & 1273093 & 28 \\
\hline Atlantic Provinces* & $\mathrm{n} / \mathrm{a}$ & 343482 & $<5$ \\
\hline Nunavut & 80.6 & 11582 & 28 \\
\hline \multicolumn{4}{|l|}{ Indigenous statust } \\
\hline Indigenous overall & 8.6 & 448865 & 116 \\
\hline First Nations overall & 9.1 & 285825 & 78 \\
\hline First Nations on-reserve & 20.2 & 105445 & 64 \\
\hline Inuit & 54.3 & 21495 & 35 \\
\hline Other & 0.8 & $\mathrm{n} / \mathrm{a}$ & $<5$ \\
\hline Non-Indigenous & 0.5 & 5368180 & 84 \\
\hline \multicolumn{4}{|c|}{$\begin{array}{l}\text { *Includes Newfoundland and Labrador, Prince Edward Island, Nova Scotia and New } \\
\text { Brunswick. Fewer than five cases reported so incidence rate not calculated. } \\
\text { †Canada and province/territory-specific populations are averaged from } 2013 \\
\text { to } 2016 \text { StatsCan July } 1 \text { yearly population estimates. Indigenous Status-specific } \\
\text { populations are taken from the } 2016 \text { StatsCan Census. }\end{array}$} \\
\hline
\end{tabular}

$(\mathrm{n}=28,14 \%)$. Annual incidence rates by province or territory of residence and First Nation and Inuit status are shown in table 2. The highest rates were in Nunavut $(80.6$ cases per 100000 , greater than 80 times that of the rest of Canada combined) and Manitoba $(8.5$ cases per 100000$)$. Of the 70 children identified as First Nations, 64 (91\%) had their residence on reserve. ${ }^{12}$

Results related to exposure and previous $\mathrm{TB}$ diagnosis are shown in table 3 . A source case was identified in $73 \%$ of cases: most $(n=91,65 \%)$ were close household contacts of either a first-degree (48\%) or second-degree (37\%) relative. Twenty-five children (of whom $12(48 \%)$ were First Nation and 9 (36\%) were Inuit) had previously received TB treatment, chiefly for latent TB infection.

Selected clinical features are shown in table 4. TB was intrathoracic in 183 (92\%) patients of whom 25 (14\%) had extrathoracic involvement. The most common extrathoracic sites were the central nervous system (CNS) and peripheral lymph nodes. Miliary or disseminated disease was seen in 20 cases. Five (14\%) cases in Inuit children involved the CNS, of whom $3(60 \%)$ had received BCG vaccine. Approximately two-thirds had symptoms, most commonly cough fever and weight loss. Only 12 (6\%) children with incident TB had the classic triad of cough, weight loss and night sweats and fewer than 5 children had haemoptysis.

One hundred and sixty-five children had at least one tuberculin skin test performed of which 144 (87\%) were positive. An interferon gamma release assay test was performed in 13 children of whom $10(77 \%)$ were positive. Overall, 80 (40\%) children had at least one positive culture from any site. One hundred and forty-four children had at least one respiratory sample taken for mycobacterial culture; culture was positive in 31 of $77(40 \%)$ sputum and 31 of $74(42 \%)$ gastric aspirate samples; $55 \%$ of children with intrathoracic TB in whom a respiratory sample was obtained had negative cultures. Nineteen of $77(25 \%)$ sputa
Table 3 Key exposure, epidemiologic and past tuberculosis history data

\begin{tabular}{|c|c|c|}
\hline & Number (n) & $\%$ \\
\hline \multicolumn{3}{|l|}{ Source case $(\mathrm{N}=198)$} \\
\hline Known & 145 & 73 \\
\hline Unknown & 53 & 27 \\
\hline \multicolumn{3}{|l|}{ Source case relationship $(\mathrm{N}=128)$} \\
\hline First-degree relative & 62 & 48 \\
\hline Second-degree relative & 47 & 37 \\
\hline Friend & 11 & 9 \\
\hline Other & 8 & 6 \\
\hline \multicolumn{3}{|l|}{ Exposure type $(\mathrm{N}=140)$} \\
\hline Close household & 91 & 65 \\
\hline Close non-household & 34 & 24 \\
\hline Casual & 6 & 4 \\
\hline Visit to endemic country & 7 & 5 \\
\hline Community/other & $<5$ & $\mathrm{n} / \mathrm{a}$ \\
\hline \multicolumn{3}{|c|}{ Source case case known resistance to first-line agents ${ }^{*}(\mathrm{~N}=137)$} \\
\hline Resistant & 5 & 4 \\
\hline Not resistant & 117 & 85 \\
\hline Not done or unknown & 15 & 11 \\
\hline \multicolumn{3}{|l|}{ BCG vaccination $(\mathrm{N}=188)$} \\
\hline Yest & 86 & 46 \\
\hline No & 81 & 43 \\
\hline Unknown & 21 & 11 \\
\hline \multicolumn{3}{|l|}{ Previous tuberculosis diagnoses or treatment } \\
\hline Latent tuberculosis, treated & 17 & 8.5 \\
\hline Latent tuberculosis, untreated & $<5$ & $\mathrm{n} / \mathrm{a}$ \\
\hline Active tuberculosis, treated & $<5$ & $\mathrm{n} / \mathrm{a}$ \\
\hline Window prophylaxis & $<5$ & $\mathrm{n} / \mathrm{a}$ \\
\hline Unspecified/unknown anti-tuberculosis therapy & $<5$ & $\mathrm{n} / \mathrm{a}$ \\
\hline
\end{tabular}

*All positive tuberculosis cultures in Canada undergo phenotypic drug-susceptibility testing. First-line agents are isoniazid, rifampin, pyrazinamide and ethambutol. tFor all children for whom immunisation status was known, BCG was received by $79 \%$ of Inuit children, $65 \%$ of First Nations children and $87 \%$ of foreign-born children.

were in those younger than 5 years; of these, fewer than 5 were culture positive.

Resistance to at least one drug was reported in $5(7 \%)$ isolates. Most were resistant to isoniazid and rifampin and thus classified as multi-drug resistant. There were no proven cases of extensively-drug-resistant TB.

The median duration of treatment for all cases was 26 weeks (IQR 24-32), for TB meningitis 48 weeks (IQR 30-65) and for disseminated disease 48 weeks (IQR 40.5-54). Median duration of therapy for the five cases with documented resistance was 48 weeks (IQR 36-48). Most children were treated with a threedrug regimen of isoniazid, rifampicin and pyrazinamide $(n=99$, $50 \%)$ or four-drug regimen that included ethambutol $(n=79$, $40 \%)$. Use of directly observed therapy was reported in 183 (96\%) cases for which this information was available.

Reported and minimum estimated rates of selected clinical events are shown in online supplemental table 1. Fifty-one per cent of children were admitted to hospital for a median of 14 days (IQR 7-19, maximum 252). Intensive care unit (ICU) admission status was known for 84 children of whom 11 (5.5\%) required ICU level care. Seven (54\%) children admitted to ICU had CNS involvement.

In 17 cases (9\%), an ADR required a change in treatment and 6 cases required hospitalisation for the ADR. The most 
Table 4 Selected clinical features and results of investigations

Number (n), n (\%) Duration, median (IQR)

\begin{tabular}{|ll|}
\hline Disease site & \\
\hline Only intrathoracic & $158(81)$ \\
\hline Intrathoracic and extrathoracic & $25(13)$ \\
\hline Only extrathoracic & $13(7)$ \\
\hline Extrathoracic sites & \\
\hline Central nervous system & $16(8)$ \\
\hline Peripheral lymph nodes & $13(7)$ \\
\hline Symptomst & \\
\hline Cough & $84(42)$ \\
\hline Fever & $52(26)$ \\
\hline Weight loss & $36(18)$ \\
\hline Haemoptysis & $<5$ \\
\hline No recorded symptoms & $65(33)$ \\
\hline Tests of infection & \\
\hline TST positive & $144 / 165(87)$ \\
\hline IGRA positive & $10 / 13(77)$ \\
\hline Positive TB culture & \\
\hline Any site (one or more) & $80 / 200(40)$ \\
\hline Sputum & $31 / 77(40)$ \\
\hline Gastric aspirates & $31 / 74(42)$ \\
\hline
\end{tabular}

* Four cases with incomplete data excluded from analysis of disease site. tNo data on presence or absence of symptoms for 18 cases.

IGRA, Interferon gamma release assay; TB, tuberculosis; TST, tuberculin skin test.

common drugs implicated in ADR were pyrazinamide $(5 \%$, most commonly for hepatotoxicity) and isoniazid (3\%, most commonly for hepatotoxicity). Other implicated drugs included amikacin (hearing loss), rifampicin (hepatotoxicity), ethambutol (visual changes), linezolid (marrow suppression) and cycloserine (neuropathy).

Longer term follow-up information was provided in 106 cases and of these, 97\% and 90\% had shown clinical and radiologic improvement, respectively, at the time of final follow-up. Sixteen cases reported an extension of therapy beyond the originally planned time frame. Most common reasons for extended treatment included poor adherence to therapy $(n=5)$, extrathoracic site of infection found after initiation of therapy $(<5)$, poor radiologic or clinical response $(<5)$ and drug resistance $(<5)$. Fewer than five cases were reported as lost to follow-up and there were no TB-related deaths.

To understand the representativeness of our cases, we compared our results to the numbers of cases reported to the PHAC during the study time frame. Cases are de-identified in both reporting systems and could not be matched. For 2014 and 2015, where reporting periods were identical, CPSP reports were $74 \%$ of all cases reported to PHAC and 90\% of PHAC reported cases when the province of Québec was excluded (online supplemental table 2). During the study, there were significant TB outbreaks in Nunavik, Québec, an Inuit region where TB is managed by clinicians not included in the CPSP surveillance system. ${ }^{13}$ Using additional demographic data from the province of Québec via PHAC (online supplemental table 3 ), we estimated a maximum number of 269 cases of childhood TB in Canada over the study period. ${ }^{14}$ Using these data, the minimum national incidence rates were 10.2/100 000/year for all Indigenous children, 100.8/100 000/year for Inuit children and 1.55/100 000/year for all children in Canada (online supplemental table 4).

\section{DISCUSSION}

This study is one of the first nation-wide surveys to collect TB data directly from treating clinicians. The overall rate of reported cases of childhood TB in this study was 1.2 per 100000 . This compares with an approximate incidence of rate of 1.6/100 000 from PHAC data ${ }^{15}$ and 1.9 in the Netherlands, ${ }^{16} 2.0$ in the $\mathrm{UK}^{17}$ and 0.7 per 100000 in the USA. ${ }^{18}$

There was wide variation across Canada geographically and by population origin of the cases. The most striking finding is the burden of TB in Indigenous children. In 2016, Indigenous people comprised $19 \%$ of all reported TB cases in all ages. ${ }^{15}$ Though Indigenous children accounted for less than $10 \%$ of Canada's childhood population, $58 \%$ of reported TB was in this group. The rates in First Nations children (9.1 per 100000 calculated from CPSP reported cases), especially those residing on reserve (20.2 per 100000$)$ are the drivers for childhood TB in the provinces of Manitoba, which had the greatest absolute number of cases, and Saskatchewan. The rate in Inuit children $(54.3 / 100$ 000 population) is similar to reported childhood TB rates in high TB burden international settings. ${ }^{1}$ Furthermore, when Québec cases not reported to CPSP are included, the minimum incidence rate in Inuit children is likely greater than 100/100 000 per year. Additionally, five cases from Newfoundland and Labrador where 90\% of PHAC reported cases are Inuit were reported to PHAC but not to the CPSP.

In recognition of this hugely disproportionate burden in Inuit peoples, there has been a recent focus by the national representative organisation of Inuit peoples (Inuit Tapiriit Kanatami) and the Federal Government on social determinants of health and access to care as major contributors to TB in Inuit peoples ${ }^{18}$ and a pledge to reduce rates in Inuit Nunangat (the traditional territory of Inuit peoples in Canada) by $2030 .{ }^{19}$ A similar strategy and monitoring programme has been implemented by the Assembly of First Nations to focus on TB on First Nation reserves. ${ }^{19}$ The disproportionate burden of TB in Indigenous people in Canada has origins in the social determinants of health, historical trauma, the residential school system and racism within the healthcare system. ${ }^{20}$ Reducing and eliminating TB in Indigenous communities through Indigenous-led and community-relevant approaches are the most urgent priorities for achieving elimination of childhood TB in Canada.

In keeping with other studies, most cases occurred in children under 5 years of age, reflecting the unique vulnerability of young children to $\mathrm{TB}^{21}$ There was a known TB contact in $82 \%$ of Canadian-born children, reinforcing the importance of contact tracing and a public health infrastructure to diagnose and prevent TB in Canada. Only 18\% of foreign-born children had a known contact; most of them likely acquired TB infection in the country of birth or by travel to a higher TB endemic country after arrival in Canada.

Presenting symptoms were non-specific but most children were symptomatic for more than 2 weeks. Haemoptysis and the classic triad of weight loss, fever and night sweats was uncommon. This study highlights acuity and health system costs of childhood TB. About half of children were hospitalised and 5.5\% were known to be admitted to ICU. TB meningitis, a condition associated with poor outcomes ${ }^{22}$ was the most common extrapulmonary site occurring in $8 \%$ of cases overall and $14 \%$ of reported disease in Inuit children. Of the children with meningitis with known BCG status, 7 (54\%) had received the BCG vaccine.

A strength of this study was inclusion of clinicians managing cases through a cross-Canada collaborative network. While monthly surveys of clinicians are labour-intensive, it is possible to obtain rich epidemiologic and clinical data that is not possible 
through public health reporting. However, it has several limitations including voluntary reporting with some cases not captured. The largest discrepancy between the reported number of CPSP and PHAC cases was in Québec. Due to under-reporting, the representativeness of the acuity of cases cannot be confirmed, though minimum rates have been estimated. Follow-up data was less well reported than the initial incident case. Reasons for missing follow-up data included the reporting physician was not the physician who provided longer term care, the physician did not consent to being recontacted or the physician did not respond. Due to CPSP privacy rules, results with size $<5$ cannot be shown, which limits the description of rare outcomes. We captured region of residence and of diagnosis, however, these may not reflect the region of disease acquisition. We are not able to determine which cases were identified via a symptomatic child presenting to care versus via contact tracing nor were we able to determine clinical details of prior TB infection. Our surveillance asked only which drugs TB isolates were resistant to, not all drugs tested. The determination of $\mathrm{TB}$ acquired during travel used dates of travel to an endemic country, contact history and onset of disease, however, infection may have been acquired at another time. We recommend that future national surveillance and statistics about childhood TB should include detailed data about indigenous status, source case characteristics, prior infection and travel.

\section{CONCLUSION}

This surveillance study identified 200 incident cases of childhood TB over 3 years. Acuity of illness and resource utilisation were high. Most cases had a known source case. Outbreaks of childhood TB in low TB burden countries are best prevented by prompt identification of source cases, and evaluation and treatment of their contacts and require a well-functioning public health infrastructure and good contact tracing. Key geographies and groups have very high incidence rates of childhood TB that are comparable with highly endemic countries. Strong community and public health engagement are required to reduce disparities and achieve elimination of childhood TB in Canada.

\footnotetext{
Author affiliations

${ }^{1}$ Infectious Diseases, The Hospital for Sick Children, Toronto, Ontario, Canada

2University of Toronto, Toronto, Ontario, Canada

${ }^{3}$ The Hospital for Sick Children, Toronto, Ontario, Canada

${ }^{4}$ University of Manitoba, Winnipeg, Manitoba, Canada

${ }^{5}$ Saskatchewan Infectious Disease Care Network, Saskatoon, Saskatchewan, Canada

${ }^{6}$ Government of Nunavut, Iqaluit, Nunavut, Canada

${ }^{7}$ Pediatric Infectious Diseases, CHU Sainte-Justine, Montreal, Quebec, Canada

${ }^{8}$ Division of Respiratory Medicine, Montreal Children's Hospital, McGill University

Health Centre, Montreal, Quebec, Canada

${ }^{9}$ University of Alberta, Edmonton, Alberta, Canada

${ }^{10}$ Alberta Health Services, Edmonton, Alberta, Canada

${ }^{11}$ British Columbia Centre for Disease Control, Vancouver, British Columbia, Canada

${ }^{12}$ The University of British Columbia, Vancouver, British Columbia, Canada

${ }^{13}$ University of Calgary, Calgary, Alberta, Canada

${ }^{14}$ London Health Sciences Centre Children's Hospital, London, Ontario, Canada

${ }^{15}$ Public Health Agency of Canada, Ottawa, Ontario, Canada

${ }^{16}$ Department of Pediatrics, McMaster University, Hamilton, Ontario, Canada

${ }^{17}$ British Columbia Women's Hospital and Health Centre, Vancouver, British

Columbia, Canada

${ }^{18}$ Childrens Hosp Eastern Ontario, Ottawa, Ontario, Canada

${ }^{19}$ Qikiqtani General Hospital, Iqaluit, Nunavut, Canada

${ }^{20}$ Ottawa Hospital Research Institute, Ottawa, Ontario, Canada

${ }^{21}$ University of Ottawa, Ottawa, Ontario, Canada

${ }^{22}$ Saskatchewan Health Authority, Saskatoon, Saskatchewan, Canada

${ }^{23}$ University of Saskatchewan, Saskatoon, Saskatchewan, Canada

${ }^{24}$ Health Canada, Ottawa, Ontario, Canada

${ }^{25}$ Dalhousie University, Halifax, Nova Scotia, Canada

${ }^{26}$ IWK Health Centre, Halifax, Nova Scotia, Canada
}

${ }^{27}$ Stanton Territorial Hospital, Yellowknife, Northwest Territories, Canada

${ }^{28}$ Toronto Public Health, Toronto, Ontario, Canada

Acknowledgements The authors wish to thank the paediatricians, paediatric subspecialists and health professionals who voluntarily respond to CPSP surveys. The authors also wish to thank members of the CPSP Steering Committee who oversee the programme and the managers and staff of the CPSP who implement the programme. The authors also wish to thank Alainna Jamal and Aaryn MontgomerySong for their assistance with database entry. This study was supported by The Public Health Agency of Canada through the CPSP and a grant from Paediatric Consultants, Hospital for Sick Children.

Contributors SKM and IK conceived the study and wrote the original protocol. SKM wrote the first draft of the manuscript. RJPG conducted statistical analyses. All authors played a role in data collection, critically revised the manuscript and approved the final version

Funding This work was funded by the Public Health Agency of Canada through the Canadian Paediatric Surveillance Program and a grant from Pediatric Consultants, Hospital for Sick Children. SKM was the recipient of a Career Development Award from the Canadian Child Health Clinician Scientist Program (CCHCSP). RJPG received funding from the SickKids Social Pediatrics Program.

Competing interests JML holds the CIHR-GSK Chair in Pediatric Vaccinology at Dalhousie University.

\section{Patient consent for publication Not required.}

Ethics approval Ethics approval was obtained from lead institution The Hospital for Sick Children (REB\#1000037963), Public Health Agency of Canada (REB\#2013-0023), and select institutions of study co-investigators that required ethical approval (University of British Columbia (REB\#H14-00851), University of Alberta (REB\#Pro00040274) and University of Saskatchewan (REB\#14-120).

Provenance and peer review Not commissioned; externally peer reviewed.

Data availability statement Data are available upon reasonable request. Deidentified data abiding by Public Health Agency of Canada surveillance privacy rules are available upon reasonable request to the corresponding author.

Supplemental material This content has been supplied by the author(s). It has not been vetted by BMJ Publishing Group Limited (BMJ) and may not have been peer-reviewed. Any opinions or recommendations discussed are solely those of the author(s) and are not endorsed by BMJ. BMJ disclaims all liability and responsibility arising from any reliance placed on the content. Where the content includes any translated material, BMJ does not warrant the accuracy and reliability of the translations (including but not limited to local regulations, clinical guidelines, terminology, drug names and drug dosages), and is not responsible for any error and/or omissions arising from translation and adaptation or otherwise.

\section{ORCID iDs}

Shaun K Morris http://orcid.org/0000-0001-9809-0822

Jeffrey M Pernica http://orcid.org/0000-0002-4380-5402

\section{REFERENCES}

1 WHO. Global tuberculosis report 2020. Geneva: World Health Organization, 2020 https://www.who.int/tb/publications/global report/TB20 Exec Sum_20201014.pdf

2 Lafreniere M, Hussain H, He N. Tuberculosis in Canada. Lancet 1946; $1: 585$.

3 Canadian-Tuberculosis-Standards-7Th-Edition @ Www.Canada.Ca. Available: https:// www.canada.ca/en/public-health/services/infectious-diseases/canadian-tuberculosisstandards-7th-edition.htm

4 Kitai I, Morris SK, Kordy F, Lam R. diagnosis and management of pediatric tuberculosis in Canada. CMAJ 2017;189:E11-16.

5 Perez-Velez CM, Marais BJ. Tuberculosis in children. N Engl J Med Overseas Ed 2012;367:348-61.

6 Graham SM, Sismanidis C, Menzies HJ, et al. Importance of tuberculosis control to address child survival. Lancet 2014;383:1605-7.

7 Reuter A, Hughes J, Furin J. Challenges and controversies in childhood tuberculosis. Lancet 2019;394:967-78.

8 Graham SM, Ahmed T, Amanullah F, et al. Evaluation of tuberculosis diagnostics in children: 1. proposed clinical case definitions for classification of intrathoracic tuberculosis disease. consensus from an expert panel. J Infect Dis 2012;205 Supp 2:S199-208.

9 Graham SM, Cuevas LE, Jean-Philippe P, et al. Clinical case definitions for classification of intrathoracic tuberculosis in children: an update. Clin Infect Dis. 2015:61:S179-87.

10 Statistics Canada. Population estimates on July 1st, by age and sex [Internet], 2018 Available: https://www150.statcan.gc.ca/t1/tbl1/en/tv.action?pid=1710000501\& pickMembers $\% 5 B 0 \% 5 D=1.15 \&$ pickMembers $\% 5 B 1 \% 5 D=2.1$ [Accessed 15 May 2019] 
11 Statistics Canada. Aboriginal Population Profile, 2016 Census [Internet], 2016. Available: https://www12.statcan.gc.ca/census-recensement/2016/dp-pd/abpopprof/ details/page.cfm?Lang=E\&Geo1=PR\&Code1=01\&Data $=$ Count\&SearchText $=$ Canada\&SearchType=Begins\&B1=All\&C1=All\&SEX_ID=1\&AGE_ID=2\&RESGEO_ ID=2 [Accessed 30 Aug 2019].

12 Government of Canada. Treaties and agreements [Internet], 2018. Available: https:// www.rcaanc-cirnac.gc.ca/eng/1100100028574/1529354437231 [Accessed 24 Mar 2020].

13 Dion R, Brisson M, Proulx JF, et al. Results of a population screening intervention for tuberculosis in a Nunavik village, Quebec, 2015-2016. Can Commun Dis Rep 2018;44:257-63.

14 Canadian Tuberculosis Reporting System Data Request - CTBRS 2018/2017 case and outcome data. Ottawa.

15 Vachon J, Gallant V, Siu W. Tuberculosis in Canada, 2016. Can Commun Dis Rep 2018;44:75-81.

16 Erkens CGM, de Vries G, Keizer ST, et al. The epidemiology of childhood tuberculosis in the Netherlands: still room for prevention. BMC Infect Dis 2014;14:295.
17 Mohiyuddin T, Seddon JA, Thomas HL, et al. The changing landscape of childhood tuberculosis in the United Kingdom: a retrospective cohort (2000-2015). Pediatr Infect Dis J 2019;38:470-5.

18 Centers for Disease Control and Prevention. Reported Tuberculosis in the United States, 2017 [Internet], 2018. Available: https://www.cdc.gov/tb/statistics/reports/ 2017/table4.htm

19 Assembly of First Nations. Tuberculosis (TB) [Internet], 2019. Available: http://www. afn.ca/tuberculosis-tb/ [Accessed 16 Jun 2021].

20 Jetty R. Tuberculosis among First Nations, Inuit and Métis children and youth in Canada: Beyond medical management [Internet]. Pediatrics and Child Health, 2020. Available: https://www.cps.ca/en/documents/position/tuberculosis-among-firstnations-inuit-and-metis-children-and-youth\#ref6 [Accessed 16 Jun 2021].

21 Marais BJ, Graham SM, Maeurer M, et al. Progress and challenges in childhood tuberculosis. Lancet Infect Dis 2013;13:287-9.

22 Chiang SS, Khan FA, Milstein MB, et al. Treatment outcomes of childhood tuberculous meningitis: a systematic review and meta-analysis. Lancet Infect Dis 2014;14:947-57. 